DOI: http://dx.doi.org/10.14201/0AQ02872539

\title{
DESIGN OF PLATFORMS BASED ON BLOCKCHAIN TECHNOLOGY APPLIED TO DIFFERENT USE CASES
}

\author{
Yeray MezQuita
}

BISITE Digital Innovation Hub, University of Salamanca, Edificio Multiusos

$I+D+I, 37007$, Salamanca, Spain

ORCID: 0000-0003-4813-4043

yeraymm@usal.es

Abstract: The developments of my $\mathrm{PhD}$ in this past year are shown in this article. It is studied thoroughly the possibilities and limits of the blockchain protocols when used in IoT platforms. It is commented how the scalability limits of blockchain technology affects the performance of the systems that make use of it. Also, a review of the state of the art has been carried out, pointing out how some solutions make use of a centralization process to improve response time and security of the blockchain. As future remarks, it should be studying the possibility of creating a public blockchain network with the IoT devices of the platform.

KEYwORDS: Blockchain; IoT platform; Multi-agent system; Review.

\section{Introduction}

Our society is evolving into a digitally interconnected world by attaching sensors and actuators to everyday objects used in people's daily lives. Those devices are capable to read their surroundings and interact with them in an automatic manner, optimizing activities of the daily life of people [1-12].

Different real objects can interact between themselves through internet, creating a network of interconnected devices defined as Internet of Things (IoT) [13-27]. The concept of IoT is very popular in resource optimization 
problems and highly demanded in today's industry due to its cost savings (Smart Grids, Smart Home, Smart Farming, Smart City) [28-32].

Although these possibilities make the use of this technology very attractive, they also bring with them some disadvantages [33-41]:

- Privacy: With devices that monitor virtually all of people's activities, companies that have access to that information also have access to the routine of our daily lives.

- Security: The continuous exchange of information between the devices, problems with the integrity of these data may arise, due to potential attacks from malicious actors.

The previously mentioned problems could be tackled by designing this type of systems with technology capable of ensuring the privacy of the users and the integrity of the generated data. Thanks to the data encryption protocol end-to-end, Blockchain Technology (BT) have the potential to cover the issues IoT platforms have [42-50].

The blockchain is an incorruptible digital distributed ledger of economic transactions that can be programmed to record not just financial transactions but virtually everything of value. This ledger consists of a peer-to-peer (P2P) network of nodes that keeps the information stored in a redundant way. In an IoT system, BT can be used instead of traditional databases, helping on getting rid of centralized controllers such as banks, accountants and governments [51-59].

The benefits BT brings with itself in IoT systems comes with some downsides that limits their performance:

- Storage capacity. In BT the size of the data stored in the chain is continually growing. This means that, as time passes and size grows up, the nodes require more resources.

- Consensus. The nodes of the blockchain network need to reach a consensus in order to add the next block of information to the blockchain. The common consensus protocol used in the most famous blockchains (Bitcoin and Ethereum) needs time and consumes a great quantity of resources, something that does not match the nature of IoT platforms, essentially composed of devices with low computational power and near real time responsiveness. 
- Scalability. The resources needed to get consensus in the network increase with the number of transactions carried out within the platform. A globally used platform needs a lot of time to handle that increase of activity.

Within the spectrum of blockchain technologies, there always exist an alternative that, with greater or lesser difficulty, can be implemented satisfactorily in the majority of the use cases. This article shows and discuss some of the solutions proposed for different IoT platforms of the literature, emphasizing what is proposed within the scope of my $\mathrm{PhD}$ thesis.

\section{Blockchain Technology: background}

BT has been created with the intention of replacing the current, centralized financial system. In [60-65] it is claimed that BT is capable of replacing intermediaries while ensuring the security of platforms. But, although BT offer resistance to traditional cyberattacks, as it gains widespread adoption, they are being developed new attacks specifically for hacking it [66].

Distributed Denial Of Services (DDOS) attacks are the most common. A kind of DDOS attack is the Malleability attack, produced when an attacker creates a copy of a transaction but with another ID, which makes the user spend double for it [71]. This attack occurs when a system that make use of a blockchain, like a bitcoin exchange, have flaws in the implementation of the code that allows the trading of cryptocurrencies.

The eclipse and sybil attacks have similar bases. In both, the attacker gains control of a large number of IP addresses of the network and surrounds the victim with them. In Eclipse attacks, the victim is not allowed to obtain transactions they are interested in. This kind of attacks has been successfully carried out in the Ethereum blockchain by Researchers from the University of Boston. In a Sybil attack, the victim is influenced by the voting power of the attacker nodes and the information they send to it, which makes the victim vulnerable to double spend attacks.

A $51 \%$ or majority attack occurred when a single entity owns the majority of the voting power of a network. An attacker who wants to take advantage of this condition can create a fork of the main chain with the transactions it wants to be done. The cryptocurrencies with a small network behind them 
are at risk, because it's easier for an attacker to gain that $51 \%$ of the network voting power.

The more proven a BT-based platform is against the attacks previously mentioned, the more trust the users give in the cryptocurrency it underlies. As a result of that trust its economic ecosystem will grow, which will translate into an increment of the cryptocurrency value and its market capitalization [72].

Some of the differential aspects of a BT based platform are: the consensus algorithm that the peers of the network use to add new blocks to the blockchain; the way in which the network is governed; and its capability to execute code that does or does not allow to deploy Turing-complete Smart Contracts in the blockchain.

Relating to the consensus algorithm, there is an increasing number of them and their own variations. The most widespread algorithms are the most proved, that's why they, or any of their variations, are shared by the vast majority of the cryptocurrencies [73].

In the Proof-of-Work (PoW) algorithm, to add a new block to the blockchain a cryptographic problem must be solved. The computational cost and the difficulty of solving the problem, the energy spent on searching for its solution (work) and the simplicity of verifying it, are enough reasons to encourage the nodes that wants to add new blocks (miners) not to cheat by adding illegal transactions.

Proof-of-Stake (PoS) is a consensus algorithm, in which miners take turns at adding new blocks. The probability of a miner to receive the turn to add a new block depends on the amount of coins deposited as escrow (Stake). This algorithm assumes that a node is going to be honest in order to avoid losing the escrow.

In the Practical Byzantine Fault Tolerance (PBFT) algorithm, the process of adding a new block is called a round. In each round a node is selected to propose a new block, the block needs to receive $2 / 3$ of the votes of all the nodes in the network in order to be valid.

Currently, every consensus algorithm has its own risks and vulnerabilities. For example, PoW wastes a massive amount of energy to produce new blocks. That algorithm is very limited in terms of scalability and its mining pools 
are centralized [23]. In the case of the PoS algorithm, its Nothing at a Stake theory causes to occur forks of the blockchain more frequently than with other consensus algorithms [3]. In the case of PBFT the main risk is that it is a permissioned protocol and not a truly decentralized.

Another feature allowed by many BTs, is the possibility to use self-enforcing programs that can control and supervise, in an automatic way, the conditions of non-trusted parties that interact between themselves and need to reach agreements. These programs are called smart contracts and their use, is allowed in most of the blockchain protocols, in a Turing Complete or non-Turing complete way.

In $[71]$ it is shown a thorough study of the smart contracts limits when used in real world applications: the data sources where smart contracts get the data from and make their clauses work with. That's why it is needed a reliable data source that allows smart contracts to interact with the outside world without losing the trust that users have placed in them.

To allow users trust the external data used in a smart contract, it arises the term oracles. Oracles are external agents that observe and validate external data of the real world. Oracles are centralized and can be black boxes, which makes them a new trusted intermediary betraying the security and reduced-trust model of blockchain applications.

Regarding on how an oracle interacts with the Smart Contracts of a blockchain, there can be classified in [68]:

- Software Oracles: This kind of oracles gather the information from sources like web sites or public databases. By doing that they can provide to Smart Contracts the most up-to-date information from Internet. Examples of their use is in temperature readings, current price of financial assets or public transport information.

- Hardware Oracles: This kind of oracles provides the Smart Contract with data directly from the physical world. These readings come from sensors that takes measurements from their environment.

- Outbound Oracles: These oracles do not provide the Smart Contract with data from outside, instead they provide another Smart contract with data from a previous one. For example, it can tell a wallet provider that the balance of a user has changed due to some conditions. 
- Consensus based oracles: A possible solution to a centralized oracle model is to make use of a set of oracles and giving them the capacity of reach a consensus, deciding the solution of a task in a decentralized way:

- The oracles provide a deposit in the smart contract.

- After that they send a vote with the result they have achieved independently.

- The result with the most votes is the one used in the execution of the Smart Contract.

- If a result given is not between acceptable and previously defined margins, the oracle in question loses the deposit.

In [76] it is also shown why the current legislation is not fully adapted to the evolution of blockchain technology. In addition, it is commented the necessity of the apparition of an audit system for the IoT devices and smart contracts that operates and control any BT-based platform.

\section{Solutions Review}

In [34] it has been reviewed some relevant works in the field of blockchain-based IoT systems. In that work, it has carried out a review of the papers that appeared first while making a search by the keywords «blockchain» and «IoT» in Google Scholar.

In [47] it is shown how BT can be applied to a supply chain in order to strengthen the security of the IoT devices that operates in it. In the case of supply chains, it is used BT to provide transparency and visibility to the transactions of assets made between actors within the supply chain.

In the case of any kind of IoT system, control and configuration of IoT devices can be made through a blockchain. By making use of RSA public key crypto-systems and signatures in the platform communications, it is possible to avoid attacks such as man-in-the-middle. Thanks to the use of the cryptographic mechanism of the $\mathrm{BT}$, messages IoT devices exchange are encrypted and signed with the private key of those who send them. In this use case, the public key of an IoT device is stored in the blockchain, while the private one is kept inside the device itself [32-50]. 
The most used approach in this kind of systems is the one in which the blockchain is not stored inside the IoT devices, rather used as a service from outside the IoT network. This is due to IoT devices being resources-constrained, while the use of a blockchain normally involves the use of many computational resources and bandwidth [51-63].

On the contrary presents a smart home use case scenario based on a permissioned blockchain. In that work, for each smart home of the platform is used a local private blockchain, in which registered devices of the smart home read and write data in it. There also exist an IoT device for each home, called Home Miner, that manage the addition or deletion of new devices and the public key cryptosystem. The Home Miner also is the one in charge of the interactions between different Smart Homes [64-71].

Following a similar blockchain approach as the previous one, in [70] it has been proposed a MAS that manage and control an agri-food supply chain. In that work, a permissioned blockchain based on the Ethereum protocol is deployed within the platform. The IoT devices make use of it when exchanging data, encrypting and signing it with the services provided by the blockchain platform.

\section{Current Works}

To continue the research shown in section 3, a study called Multi-Agent Architecture for Peer-to-Peer Electricity Trading based on Blockchain Technology, has been defended in the special session Blockchain Technology and its Application (BCTA) of the International Conference on Information, Communication and Automation Technologies (ICAT). In that work has been proposed a MAS-based architecture that makes use as a Service of the BT provided by the public network of Ethereum to allow peer-to-peer transactions of energy in a microgrid. The MAS is in charge of automating and optimizing the interactions between entities in this platform, following a Game-Theory model. While the BT used, have more proven security than any of the permissioned solutions proposed in the literature, at the cost of losing global scalability [72-73].

In the previous work it has been done an analysis of the costs that the users should pay to use that platform. A similar analysis has been done in 
another proposal: Beneficios de la incorporación de la tecnología blockchain en el proceso de registro de la propiedad. That work has been accepted in the International Conference on Blockchain Technology in Contracting Impact on Financial, Notary, Registry and Judicial Systems. In that work, it is shown the reduction in costs that the Spanish property register would receive if the country adapts that process to allow the use of BT within it.

Like in the articles [54] it is possible to take advantage of the distribution of task a MAS provide and optimize any BT-based system. The next step that will be taken, will be to implement a negotiation algorithm that can improve payoffs and performance of the platform proposed in the ICAT paper.

Another good way to improve the performance of BT-based systems is to make use of a blockchain deployed within the IoT devices. With that approach, it is possible to stop depending on the latency of the network to read the data stored in the blockchain, but it is needed to take into account the losing security and decentralization of similar approaches. It has been analyzed the possibility of implementing this kind of platforms in [62]. In that work it has numerated and detailed some of the problems and challenges that this kind of platforms currently have, like scalability, data privacy, latency times, and some legal aspects.

\section{Conclusions}

In this paper it has been pointed out the advantages and limitations of the use of BT in any IoT platform. Although it can be increased the security of those platforms, it also reduces their performance.

In addition, it has been studied some representative Io $\mathrm{T}$ platforms based on BT from the literature. In those solutions, it has been shown that the trend they follow is to make use of the blockchain as a service from outside the IoT network of devices.

In future works it will be studied the possibility of creating the blockchain network with the IoT devices of the platform. The aim of that solution will be to stop relying in the internet latency and try to make cheaper the use of the blockchain protocols within that platform. 


\section{Acknowledgments}

The research of Yeray Mezquita is supported by the pre-doctoral fellowship from the University of Salamanca and Banco Santander. Also, this work has been partially supported by the Salamanca Ciudad de Cultura y Saberes Foundation under the Talent Attraction Program (CHROMOSOME project).

\section{References}

1. Koetsier, J., Corchado, E., MacDonald, D., Corchado, J., \& Fyfe, C. (2004, June). Kernel maximum likelihood hebbian learning. In International Conference on Computational Science (pp. 650-653). Springer, Berlin, Heidelberg.

2. Pavón, J., \& Corchado, J. (2004). Agents for the web. International journal of Web engineering and technology, 1(4), 393-396.

3. Fdez-Riverola, F., Díaz, F., Borrajo, M. L., Yáñez, J. C., \& Corchado, J. M. (2005, August). Improving gene selection in microarray data analysis using fuzzy patterns inside a cbr system. In International Conference on Case-Based Reasoning (pp. 191-205). Springer, Berlin, Heidelberg.

4. Fernández-Riverola, F., \& Corchado, J. M. (2003, November). Employing tsk fuzzy models to automate the revision stage of a cbr system. In Conference on Technology Transfer (pp. 302-311). Springer, Berlin, Heidelberg.

5. Corchado, J. M., \& Aiken, J. (1998). Expert system for modelling water masses. In WORKSHOP ON DATA MINING. GLASGOW, SCOTLAND.

6. Corchado, J. M., \& Aiken, J. (1998). Neuro-symbolic reasoning for real time oceanographic problems. In CONFERENCE ON DATA MINING. IEE, SAVOY PLACE, LONDON.

7. Corchado, J. M. (1998). Models for integrating artificial intelligence approaches. DOCTORAL CONSORTIUM ON KNOWLEDGE DISCOVERY AND DATA MINING. PAISLEY, UK.

8. Borrajo, M. L., Corchado, J. M., Yáñez, J. C., Fdez-Riverola, F., \& Díaz, F. (2005, August). Autonomous internal control system for small to medium firms. In International Conference on Case-Based Reasoning (pp. 106-121). Springer, Berlin, Heidelberg.

9. Fdez-Riverola, F., Díaz, F., \& Corchado, J. M. (2004, November). Applying rough sets reduction techniques to the construction of a fuzzy rule base for case based reasoning. In Ibero-American Conference on Artificial Intelligence (pp. 83-92). Springer, Berlin, Heidelberg. 
10. Corchado, J., \& Lees, B. (1998). Case based reasoning opportunities and technologies. In CONFERENCE ON KNOWLEDGE DISCOVERY. IEE, SAVOY PLACE, LONDON.

11. Corchado, J., \& Lees, B. (1998). Artificial neural networks in pattern recognition: multicollinearity and heterocedasticity. In COLLOQUIUM ON KNOWLEDGE DISCOVERY. LONDON, UK.

12. Corchado, J., \& Lees, B. (1998). An overview of intelligent frameworks. In COLLOQUIUM ON INTELLIGENT SYSTEMS. IEE, LONDON, UK.

13. Corchado, J. M., \& Lees, B. (1998). Probis: Modelling intelligence with hybrid systems. In WORKSHOP ON DATA MINING. University of GLASGOW, SCOTLAND, UK.

14. Corchado, J. (1998). Real time forecast with intelligent systems. In CONFERENCE ON KNOWLEDGE DISCOVERY. IEE, SAVOY PLACE, LONDON.

15. Corchado, J. M. (1997). Bdi multiagent hybrid architecture for project management. In IEEE COLLOQUIUM ON KNOWLEDGE DISCOVERY AND DATA MINING. LONDON ENGLAND.

16. Corchado, J. M. (1997). System for decision making: a practical case. In CONFERENCE ON KNOWLEDGE DISCOVERY AND DATA MINING. IEE, LONDON, UK.

17. Corchado, J. (1995). Cbr systems, an overview. In INTERNATIONAL CONFERENCE ON INTELLIGENT SYSTEMS. LONDON, ENGLAND, UK.

18. Li, T., Sun, S., Corchado, J. M., \& Siyau, M. F. (2014, July). A particle dyeing approach for track continuity for the SMC-PHD filter. In 17 th International Conference on Information Fusion (FUSION) (pp. 1-8). IEEE.

19. Corchado, E. S., Corchado, J. M., Sáiz, L., \& Lara, A. (2004, July). A beta-cooperative cbr system for constructing a business management model. In Industrial Conference on Data Mining (pp. 42-49). Springer, Berlin, Heidelberg.

20. Corchado, J. M., \& Lees, B. (1998). Integration ai models. In WORKSHOP ON KNOWLEDGE DISCOVERY AND DATA MINING. PML-NERC, PLYMOUTHLONDON, UK.

21. Corchado, J. M., \& Lees, B. (1998). Cognitive models for integrating artificial intelligence approaches. In AII WORKSHOP ON KNOWLEDGE DISCOVERY. GLASGOW, UK.

22. Corchado, J. M. (1997). Real time forecast with intelligent systems: Cbrs and anns. In WORKSHOP ON ARTIFICIAL NEURAL NETWORKS. ABERDEEN (pp. 1-3). 
23. Casado-Vara, R., Prieto, J., De la Prieta, F., \& Corchado, J. M. (2018). How blockchain improves the supply chain: Case study alimentary supply chain. Procedia computer science, 134, 393-398.

24. Corchado, J. M. (1996). Case-base reasoning recommendation system. In IEEE COLLOQUIUM ON KNOWLEDGE DISCOVERY. LONDON, UK.

25. Corchado, J. M. (1995). Neuro-symbolic reasoning-a solution for complex problemas. In INTERNATIONAL CONFERENCE ON INTELLIGENT SYSTEMS. LONDON, UK.

26. Corchado, J. M. (1995). Multi agent tools: a case study. In IEEE COLLOQUIUM ON KNOWLEDGE DISCOVERY. LONDON ENGLAND, UK.

27. Casado-Vara, R., Prieto-Castrillo, F., \& Corchado, J. M. (2018). A game theory approach for cooperative control to improve data quality and false data detection in WSN. International Journal of Robust and Nonlinear Control, 28(16), 50875102.

28. Corchado, J. M., \& Lees, B. (2001). Adaptation of cases for case based forecasting with neural network support. In Soft computing in case based reasoning (pp. 293-319). Springer, London.

29. Rodriguez, J. M. C. (2000). Neuro-symbolic model for real-time forecasting problems (Doctoral dissertation, University of Paisley).

30. Casado-Vara, R., Chamoso, P., De la Prieta, F., Prieto, J., \& Corchado, J. M. (2019). Non-linear adaptive closed-loop control system for improved efficiency in IoT-blockchain management. Information Fusion, 49, 227-239.

31. Li, T., Sun, S., Corchado, J. M., \& Siyau, M. F. (2014, July). Random finite setbased Bayesian filters using magnitude-adaptive target birth intensity. In 17 th International Conference on Information Fusion (FUSION) (pp. 1-8). IEEE.

32. Rodríguez, S., Gil, O., De La Prieta, F., Zato, C., Corchado, J. M., Vega, P., \& Francisco, M. (2010, May). People detection and stereoscopic analysis using MAS. In 2010 IEEE 14th International Conference on Intelligent Engineering Systems (pp. 159-164). IEEE.

33. Guillén, J. H., del Rey, A. M., \& Casado-Vara, R. (2019). Security Countermeasures of a SCIRAS Model for Advanced Malware Propagation. IEEE Access, 7 , $135472-135478$.

34. Corchado, J. M. (1996). Artificial intelligence models: composed systems as a solution. In IEEE COLLOQUIUM ON KNOWLEDGE DISCOVERY. LONDON ENGLAND, UK.

35. Casado-Vara, R., De la Prieta, F., Rodriguez, S., Prieto, J., \& Corchado, J. M. (2018, June). Cooperative Algorithm to Improve Temperature Control in Recovery Unit 
of Healthcare Facilities. In International Symposium on Distributed Computing and Artificial Intelligence (pp. 49-62). Springer, Cham.

36. Corchado, J. M. (1995). Hybrid cbr system for real-time temperature forecasting in the ocean. In IEEE COLLOQUIUM ON KNOWLEDGE DISCOVERY. LONDON, UK.

37. Corchado, J. M. (1995). A distributed recommendation system assos. In IEEE COLLOQUIUM ON KNOWLEDGE DISCOVERY. IEE, LONDON, UK.

38. Corchado, J. M. (1995). The use of kernel methods in cbr systems. In INTERNATIONAL CONFERENCE ON INTELLIGENT SYSTEMS. LONDON ENGLAND UK.

39. Corchado, J. M. (1995). Case based reasoning systems: automatic construction. In INTERNATIONAL CONFERENCE ON INTELLIGENT SYSTEMS. LONDON ENGLAND UK.

40. Casado-Vara, R., de la Prieta, F., Prieto, J., \& Corchado, J. M. (2018, November). Blockchain framework for Io'T data quality via edge computing. In Proceedings of the 1st Workshop on Blockchain-enabled Networked Sensor Systems (pp. 19-24). ACM.

41. Gil, A. B., De la Prieta, F., \& Rodríguez, S. (2011). Automatic Learning Object Extraction and Classification in Heterogeneous Environments. In Highlights in Practical Applications of Agents and Multiagent Systems (pp. 109-116). Springer, Berlin, Heidelberg.

42. Rodríguez, S., Tapia, D. I., Sanz, E., Zato, C., de la Prieta, F., \& Gil, O. (2010, July). Cloud computing integrated into service-oriented multi-agent architecture. In International Conference on Information Technology for Balanced Automation Systems (pp. 251-259). Springer, Berlin, Heidelberg.

43. Casteleiro-Roca, J. L., Pérez, J. A. M., Piñón-Pazos, A. J., Calvo-Rolle, J. L., \& Corchado, E. (2015). Modeling the electromyogram (EMG) of patients undergoing anesthesia during surgery. In 10th international conference on soft computing models in industrial and environmental applications (pp. 273-283). Springer, Cham.

44. Mazuelas, S., Lorenzo, R. M., Bahillo, A., Fernández, P., Prieto, J., \& Abril, E. J. (2010). Topology Assessment Provided by Weighted Barycentric Parameters in Harsh Environment Wireless Location Systems. IEEE Transactions on Signal Processing, 58(7), 3842-3857. doi:10.1109/TSP.2010.2047394

45. Prieto, J., Alonso, A. A., la Rosa, de, R., \& Carrera, A. (2014). Adaptive Framework for Uncertainy Analysis in Electromagnetic Field Measurements. Radiation Protection Dosimetry. doi:10.1093/rpd/ncu260 
46. Prieto, J., Bahillo, A., Mazuelas, S., Blas, J., Fernández, P., \& Lorenzo, R. M. (2008). RTS/CTS mechanism with IEEE 802.11 for indoor location. Presented at the NAV08/ILA37, The Navigation Conference \& Exhibition.

47. Prieto, J., Bahillo, A., Mazuelas, S., Fernández, P., Lorenzo, R. M., \& Abril, E. J. (2012a). Self-Calibration of TOA/Distance Relationship for Wireless Localization in Harsh Environments. Presented at the 2012 IEEE International Conference on Communications (ICC 2012).

48. Prieto, J., Bahillo, A., Mazuelas, S., Lorenzo, R. M., Blas, J., \& Fernández, P. (2009a). Adding indoor location capabilities to an IEEE 802.11 WLAN using real-time RTT measurements (pp. 113-119). IEEE Press. Retrieved from http:// dl.acm.org/citation.cfm? $\mathrm{id}=1689059.1689083 \& \mathrm{coll}=\mathrm{DL} \& \mathrm{dl}=\mathrm{GUIDE} \& \mathrm{CFID}=$ 647274630\&CFTOKEN $=45917612$

49. Bullon, Juan, et al. «Manufacturing processes in the textile industry. Expert Systems for fabrics production.» ADCAIJ: Advances in Distributed Computing and Artificial Intelligence Journal 6.4 (2017): 15-23.

50. Souza de Castro, Lucas Fernando, Gleifer Vaz Alves, \& André Pinz Borges. «Using trust degree for agents in order to assign spots in a Smart Parking.» (2017).

51. Moung, Ervin. «A Comparison of the YCBCR Color Space with Gray Scale for Face Recognition for Surveillance Applications.» ADCAIJ: Advances in Distributed Computing and Artificial Intelligence Journal [Online], 6.4 (2017): 25-33.

52. Kethareswaran, V., \& C. SANKAR RAM. «An Indian Perspective on the adverse impact of Internet of Things (IoT).» ADCAIJ: Advances in Distributed Computing and Artificial Intelligence Journal 6.4 (2017): 35-40.

53. Cunha, Rafhael, Cleo Billa, \& Diana Adamatti. «Development of a Graphical Tool to integrate the Prometheus AEOlus methodology and Jason Platform.» ADCAIJ: Advances in Distributed Computing and Artificial Intelligence Journal 6.2 (2017): 57-70.

54. Crespo-Ramos, M. J., MachóN-GonzáLez, I., LóPez-GarcíA, H., \& Calvo-Rolle, J. L. (2013). Detection of locally relevant variables using SOM-NG algorithm. Engineering Applications of Artificial Intelligence, 26(8), 1992-2000.

55. Vera, Jefferson Stewart Espinosa. «Human rights in the ethical protection of youth in social networks-the case of Colombia and Peru.» ADCAIJ: Advances in Distributed Computing and Artificial Intelligence Journal 6.4 (2017): 71-79.

56. Casado-Vara, R., Martin-del Rey, A., Affes, S., Prieto, J., \& Corchado, J. M. (2020). IoT network slicing on virtual layers of homogeneous data for improved algorithm operation in smart buildings. Future Generation Computer Systems, 102, 965-977. 
57. Mateen, Abdul, et al. «Secure data access control with perception reasoning.» ADCAIJ: Advances in Distributed Computing and Artificial Intelligence Journal 7.1 (2018): 13-28.

58. Teixeira, Eduardo Porto, Eder Goncalves, \& Diana F. Adamatti. «Ulises: A AgentBased System For Timbre Classification.»ADCAIJ: Advances in Distributed Computing and Artificial Intelligence Journal 7.1 (2018): 29-40.

59. Van Haare Heijmeijer, Alexis, \& Gleifer Vaz Alves. «Development of a Middleware between SUMO simulation tool and JaCaMo framework.» ADCAIJ: Advances in Distributed Computing and Artificial Intelligence Journal 7.2: 5-15.

60. Glaeser, Stefania da Silveira, et al. «Modeling of Circadian Rhythm under influence of Pain: an approach based on Multi-agent Simulation.» ADCAIJ: Advances in Distributed Computing and Artificial Intelligence Journal 7.2 (2018): 17-25.

61. de Melo, Maximilian Jaderson, et al. «Robust and adaptive chatter free formation control of wheeled mobile robots with uncertainties.» ADCAIJ: Advances in Distributed Computing and Artificial Intelligence Journal 7.2 (2018): 27-42.

62. Ribeiro, Catarina, et al. "Customized normalization clustering meth-odology for consumers with heterogeneous characteristics.» ADCAIJ: Advances in Distributed Computing and Artificial Intelligence Journal 7.2 (2018): 53-69.

63. Becerril, Anahiby Anyel. «The value of our personal data in the Big Data and the Internet of all Things Era.» ADCAIJ: Advances in Distributed Computing and Artificial Intelligence Journal 7.2 (2018): 71-80.

64. Garcia, Ana Cristina Bicharra, \& Adriana Santarosa Vivacqua. «ACoPla: a Multiagent Simulator to Study Individual Strategies in Dynamic Situations.» ADCAIJ: Advances in Distributed Computing and Artificial Intelligence Journal 7.2: 81-91.

65. Casado-Vara, R., Novais, P., Gil, A. B., Prieto, J., \& Corchado, J. M. (2019). Distributed continuous-time fault estimation control for multiple devices in IoT networks. IEEE Access, 7, 11972-11984.

66. Jörg Bremer, Sebastian Lehnhoff. (2017) Decentralized Coalition Formation with Agent-based Combinatorial Heuristics. ADCAIJ: Advances in Distributed Computing and Artificial Intelligence Journal (ISSN: 2255-2863), Salamanca, v. 6, n. 3

67. Rafael Cauê Cardoso, Rafael Heitor Bordini. (2017) A Multi-Agent Extension of a Hierarchical Task Network Planning Formalism. ADCAIJ: Advances in Distributed Computing and Artificial Intelligence Journal (ISSN: 2255-2863), Salamanca, v. 6, n. 2

68. Enyo Gonçalves, Mariela Cortés, Marcos De Oliveira, Nécio Veras, Mário Falcão, Jaelson Castro (2017). An Analysis of Software Agents, Environments and Applications School: Retrospective, Relevance, \& Trends. ADCAIJ: Advances in 
Distributed Computing and Artificial Intelligence Journal (ISSN: 2255-2863), Salamanca, v. 6, n. 2

69. Eduardo Porto Teixeira, Eder M. N. Goncalves, Diana F. Adamatti (2017). Ulises: A Agent-Based System For Timbre Classification. ADCAIJ: Advances in Distributed Computing and Artificial Intelligence Journal (ISSN: 2255-2863), Salamanca, v. 6, n. 2

70. Lucas Fernando Souza de Castro, Gleifer Vaz Alves, André Pinz Borges (2017). Using trust degree for agents in order to assign spots in a Smart Parking. ADCAIJ: Advances in Distributed Computing and Artificial Intelligence Journal (ISSN: 2255-2863), Salamanca, v. 6, n. 2

71. Aversa, R., Petrescu, R. V., Akash, B., Bucinell, R., Corchado, J., Berto, F., ... \& Petrescu, F. I. (2017). Kinematics and forces to a new model forging manipulator. American Journal of Applied Sciences, 14(1), 60-80.

72. Li, T., Sun, S., Bolić, M., \& Corchado, J. M. (2016). Algorithm design for parallel implementation of the SMC-PHD filter. Signal Processing, 119, 115-127.

73. Corchado, J. M., Aiken, J., Corchado, E. S., \& Fdez-Riverola, F. (2005). Evaluating the air-sea interactions and fluxes using an instance-based reasoning system. AI Communications, 18(4), 247-256. 
